\title{
Research on Semantic Programming Language
}

\author{
Shi Ying \\ State Key Laboratory of Software Engineering, \\ Wuhan University, Wuhan, China \\ yingshi@whu.edu.cn
}

\begin{abstract}
As technologies of Semantic Web Service are gradually matured, developing intelligent web applications with Semantic Web Services becomes an important research topic in Software Engineering. This speech introduces our efforts on Semantic Web Service oriented programming. Employing the concept of semantic computing into service-oriented programming, we proposed a programming language SPL, Semantic Programming Language, which supports the expression and process of semantic information. Based on collaboration of semantic space and information space, the running mechanism of SPL program is presented, which provides SPL program with higher flexibility and stronger adaptability to changes. Furthermore, with the introduction of semantic operators, a kind of searching conditional expression is offered to facilitate the search of Semantic Web Services with greater preciseness and higher flexibility. Besides, semantic based policy and exception mechanism are also brought in to improve the intelligence of policy inference and exception handing in SPL program. At the same time, a platform that supports design and running of SPL program is developed.
\end{abstract}

